\title{
Spin-Wave Dispersion Measurement by Variable-Gap Propagating Spin-Wave Spectroscopy
}

\author{
Marek Vaňatka $\odot,{ }^{1, *}$ Krzysztof Szulc $\odot,{ }^{2}$ Ondřej Wojewoda $\odot,{ }^{1}$ Carsten Dubs $\odot,{ }^{3}$ Andrii V. Chumak $\odot,{ }^{4}$ \\ Maciej Krawczyk, ${ }^{2}$ Oleksandr V. Dobrovolskiy $\odot,{ }^{4}$ Jarosław W. Kłos, ${ }^{2}$ and Michal Urbánek ${ }^{1,5, \dagger}$ \\ ${ }^{1}$ CEITEC BUT, Brno University of Technology, 61200 Brno, Czech Republic \\ ${ }^{2}$ ISQI, Faculty of Physics, Adam Mickiewicz University, 61-614 Poznań, Poland \\ ${ }^{3}$ INNOVENT e.V. Technologieentwicklung, 07745 Jena, Germany \\ ${ }^{4}$ Faculty of Physics, University of Vienna, 1090 Vienna, Austria \\ ${ }^{5}$ Institute of Physical Engineering, Brno University of Technology, 61669 Brno, Czech Republic
}

(Received 23 July 2021; revised 13 October 2021; accepted 20 October 2021; published 17 November 2021)

\begin{abstract}
Knowledge of the spin-wave dispersion relation is a prerequisite for the explanation of many magnonic phenomena as well as for the practical design of magnonic devices. Spin-wave dispersion measurement by established optical techniques such as Brillouin light scattering or the magneto-optical Kerr effect at ultralow temperatures is often forbiddingly complicated. By contrast, microwave spectroscopy can be used at all temperatures but it usually lacks spatial and wave-number resolution. Here we develop a variablegap-propagating-spin-wave-spectroscopy (VGPSWS) method for the deduction of the dispersion relation of spin waves in a wide frequency and wave-number range. The method is based on the phase-resolved analysis of the spin-wave transmission between two antennas with variable spacing, in conjunction with theoretical data treatment. We validate the method for in-plane magnetized Co-Fe-B and yttrium iron garnet thin films in $\mathbf{k} \perp \mathbf{B}$ and $\mathbf{k} \| \mathbf{B}$ geometries by deducing the full set of material and spin-wave parameters, including spin-wave dispersion, hybridization of the fundamental mode with the higher-order perpendicular standing spin-wave modes, and surface spin pinning. The compatibility of microwaves with low temperatures makes this approach attractive for cryogenic magnonics at the nanoscale.
\end{abstract}

DOI: 10.1103/PhysRevApplied.16.054033

\section{INTRODUCTION}

The properties of magnetic materials are of great interest due to several application concepts regarding, for example, memories, sensors, microwave devices, or logic devices [1]. In the emerging field of magnonics, which uses spin waves for data transport and processing, the essential system characteristic is the spin-wave dispersion relation. It provides a connection between the wave-vector space and frequency space, and it also dictates other properties, such as the group velocity $v_{g}$ and decay length $\Lambda$. In thin films (approximately less than $30 \mathrm{~nm}$ ), only the fundamental mode is observed when the experimentally accessible frequency range is limited to a few gigahertz. In contrast, the spin-wave dispersion of thicker films can be

*marek.vanatka@live.com

†michal.urbanek@ceitec.vutbr.cz

Published by the American Physical Society under the terms of the Creative Commons Attribution 4.0 International license. Further distribution of this work must maintain attribution to the author(s) and the published article's title, journal citation, and DOI. rather complex as multiple perpendicular standing modes may appear in the spectrum, exhibiting frequency crossing and hybridization [2]. The spin-wave dispersion measurement is typically done by wave-vector resolved [2,3] or phase-resolved [4,5] Brillouin light scattering (BLS). Current interest in quantum computing, quantum magnonics [6], and superconductor-ferromagnet hybrid systems $[7,8]$ increases the need for material characterization at ultralow temperatures, where optical access is typically extremely complicated. All-electrical measurements are usually preferable in these applications.

Spin-wave dispersion measurement is also possible by means of propagating spin-wave spectroscopy (PSWS). PSWS is a technique that uses a vector network analyzer (VNA) connected to a pair of microwave antennas [e.g., striplines or coplanar waveguides (CPWs)] by microwave probes $[9,10]$. The two antennas (i.e., spin-wave transmitter and receiver) have a gap between them over which the spin waves propagate, as shown in Fig. 1(a). The transmitting antenna is powered by the VNA's microwave source, and the receiving antenna serves as an induction pickup detected by the VNA's second port. The antenna type determines the excitation properties and can 


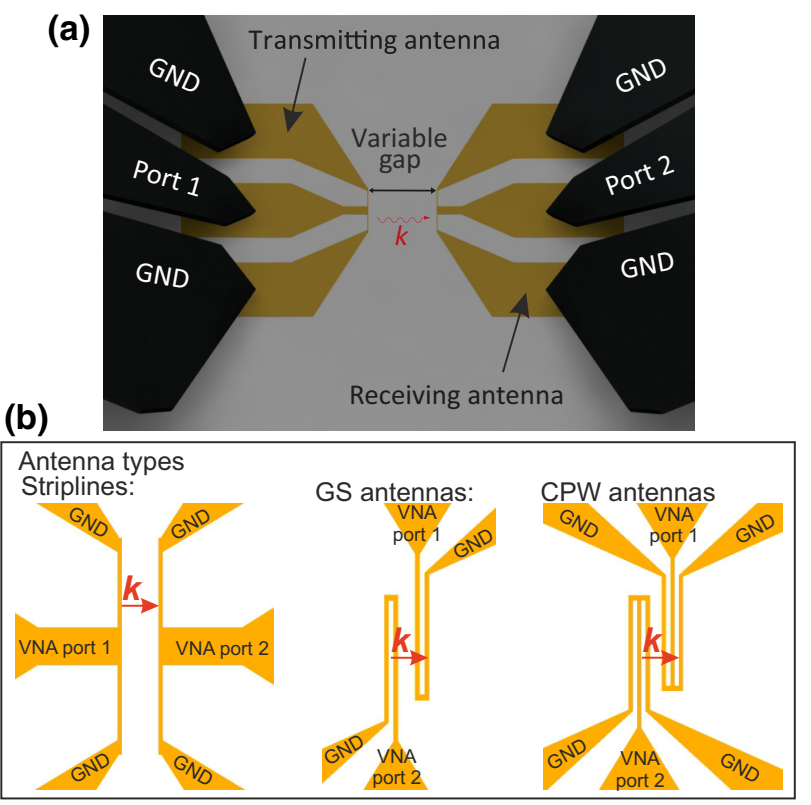

FIG. 1. (a) Schematics of the PSWS experiment using a pair of stripline antennas connected to the VNA by microwave probes. (b) Schematic geometry of the three antenna types used in the experiments. GND, ground.

be adjusted to the experiment. The three main types of antennas used in experiments are striplines (rectangular wires), U-shaped ground-signal (GS) antennas, and CPW antennas. The schematic geometry of all three antenna types is shown in Fig. 1(b). Striplines provide a continuous spectrum where the maximum excited wave-vector $k$ is limited by the stripline width. Ciubotaru et al. [11] showed scalability of the antennas, where 125 -nm-wide striplines provided a wide continuous $k$-vector band. GS antennas (e.g., Refs. [9,12,13]) and CPWs (e.g., Refs. [14-17]) are good alternatives, both providing a filtering capability for the $k$-vector spectrum allowing only specific ranges to exist. PSWS can be used on both nanostructured materials (stripes) [11-15,18-20] and layers [17,21]. It was previously shown that the PSWS signal can be negligibly different for continuous layers and wide stripes [17]. PSWS signals can also be modeled [15,17,22].

In previous reports $[17,18]$, the spin-wave dispersion was extracted from PSWS spectra measured on yttrium iron garnet (YIG) by means of CPW excitation. As the CPW excitation spectrum exhibits distinct peaks in $k$ space, it allows the extraction of one point in the spin-wave dispersion for each peak. The central $k$-vector of each peak is then assigned to a frequency either from the envelope of the $S_{21}$ sweep [18,23] or by fitting the $S_{21}$ spectrum [17]. This approach is limited to only several extracted points, and it is not easily transferable to metallic materials because of the low signal amplitude (compared with YIG) caused by large damping, making it impossible to use more than two peaks from the CPW antenna's excitation spectrum.

Here we show that spin-wave dispersion measurement using a VNA is possible with a high level of detail determined by the VNA frequency step.

\section{MEASUREMENT SETUP AND SAMPLE PREPARATION}

Our setup is based on a Rohde \& Schwarz ZVA50 VNA and GGB Industries microwave probes to establish a connection to the two antennas lithographically fabricated on top of magnetic thin films. The samples are placed in a gap of a rotatable electromagnet, allowing one to apply an inplane magnetic field of up to $400 \mathrm{mT}$ in an arbitrary direction with respect to the spin-wave propagation direction. The setup operates at room temperature; however, transfer of the experiment to a millikelvin cryostat should also be possible, as suitable microwave-probe solutions have already been developed [24]. The antenna fabrication consists of the electron-beam patterning of the antenna shapes into a PMMA resist and the electron-beam evaporation of a $\operatorname{Ti}(5 \mathrm{~nm}) / \mathrm{Cu}(85 \mathrm{~nm}) / \mathrm{Au}(10 \mathrm{~nm})$ multilayer followed by lift-off. The antennas are placed directly on the magnetic films (the 5-nm Ti adhesion layer also acts as sufficient insulation between the antenna and the conductive $\mathrm{Co}-\mathrm{Fe}-$ B film). Co-Fe-B films with nominal thicknesses of 30 and $100 \mathrm{~nm}$ are magnetron-sputtered from a $\mathrm{Co}_{40} \mathrm{Fe}_{40} \mathrm{~B}_{20}$ (with proportions given in atom percent) target onto a GaAs(100) substrate with a $5 \mathrm{~nm}$-thick Ta buffer layer. The YIG(111) films are grown by liquid-phase epitaxy on top of a $500-\mu \mathrm{m}$-thick gadolinium gallium garnet (111) substrate $[25,26]$. The VNA is set with use of a calibration substrate supplied with the microwave probes. A power sweep is performed before measurement of each sampleantenna combination to find a suitable power level to avoid nonlinear phenomena [27] and maintain a sufficient signal-to-noise ratio.

The VNA controls and analyses the electric signals of the transmitting and receiving antennas in both the amplitude domain and the phase domain; therefore, it can measure the phase Arg acquired by the spectral components of the spin wave while it propagates between antennas. Our analysis is based on the $S_{21}$ transmission parameter. The transmitted spin-wave signal is modified by a nonmagnetic background $S_{21}^{\text {background }}$ that is always present in the experiment due to direct electromagnetic crosstalk between the antennas. This background is constant for different values of the static magnetic field, and therefore it is possible to evaluate it as the median over all measured magnetic fields $S_{21}^{\text {background }}(f)=\operatorname{Med}\left[S_{21}(B, f)\right]$. The subtracted signal $\Delta S_{21}$ is then calculated as $\Delta S_{21}(B, f)=S_{21}(B, f)-$ $S_{21}^{\text {background }}(f)$. 
Figures 2(a) and 2(b) show the raw $S_{21}$ signal and Figs. 2(c) and 2(d) show the $\Delta S_{21}$ signal after nonmagnetic background subtraction. The data are measured at a power output of $0-\mathrm{dBm}$ for a $30-\mathrm{nm}$-thick Co-Fe-B film over the 1.8- $\mu \mathrm{m}$ gap in the $\mathbf{k} \perp \mathbf{B}$ geometry (magnetostatic surface waves) and with 500 -nm-wide striplines used as excitation and detection antennas. This geometry is known to be nonreciprocal with an exponential distribution of the dynamic magnetization along the layer's thickness due to the surface localization of the mode [28,29]. The higher signal amplitude in the $+B$ part of the spectrum is caused by both the stronger excitation and the larger induction pickup from spin waves propagating at the nearer surface. To achieve the best result, we focus on the $+B$ part of the spectrum (or alternatively on the $-B$ part and use the reverse transmission parameter $S_{12}$ ). Figure 2(e) shows a plot of the real and imaginary parts of $\Delta S_{21}$ measured at $20 \mathrm{mT}$. The corresponding phase is shown in Fig. 2(f). The phase is unwrapped by removing its artificial $2 \pi$ jumps to obtain a continuous dependence. The phase rises from the ferromagnetic resonance frequency until it reaches the
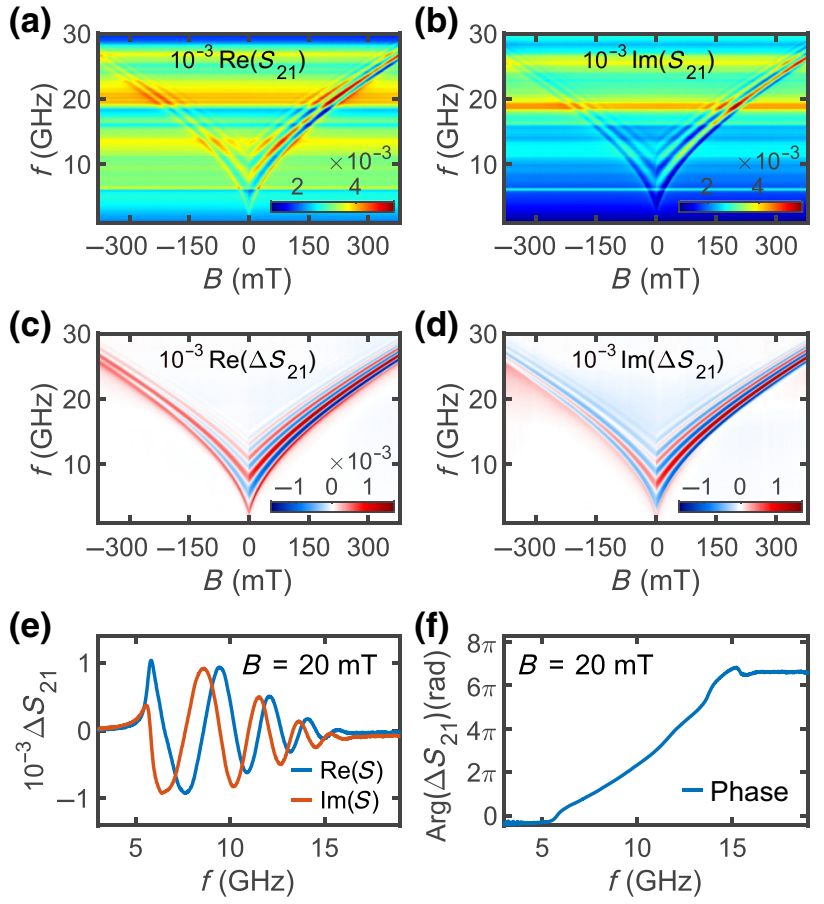

FIG. 2. The outcomes of the PSWS measurement for a 30-nmthick Co-Fe-B layer in the $\mathbf{k} \perp \mathbf{B}$ geometry and for a gap width of $1.8 \mu \mathrm{m}$. Real (a) and imaginary (b) parts of $S_{21}(B, f)$ as measured by the VNA. Real (c) and imaginary (d) parts of $\Delta S_{21}(B, f)$, obtained by subtraction of the nonmagnetic background from $S_{21}(B, f)$. The measurement reveals the nonreciprocity of spinwave propagation for the $\mathbf{k} \perp \mathbf{B}$ geometry, which is reflected in the larger signal in $+B$ fields than in $-B$ fields. (e) Real and imaginary parts of $\Delta S_{21}(f)$ and (f) the unwrapped phase of $\Delta S_{21}(f)$ at fixed magnetic field $B=20 \mathrm{mT}$. antenna's excitation limits. Beyond this point, the signal loses its coherency due to an insufficient signal-to-noise ratio, and therefore the phase stops evolving. The slope of the phase $\phi$ in the frequency domain depends on the gap width $D[\phi(f)=k(f) D]$; it changes more rapidly for wider gaps.

In the next step, we repeat the measurement on multiple instances of identical antenna structures (with different gap width) prepared on the same 30-nm Co-Fe-B thin film sample. The phases measured over 11 gap widths are shown in Fig. 3(a). The phases are on the same level before the frequency reaches ferromagnetic resonance, and then they start to rise, because spin waves start to propagate and accumulate phase over the distance traveled. At a fixed frequency, the smallest (the largest) amount of the phase is acquired for the narrowest (the widest) gap, which corresponds to the lowest (the uppermost) curve. Then we project the measured phases into a phase-versus-gap-width plot [selected frequencies are plotted in Fig. 3(b)]. In this projection, the phase shows a linear dependence (for a coherent plane wave) that can be fitted. The slope (i.e., the phase acquired per unit distance) equals, by definition, the $k$-vector at the given frequency. We then plot the extracted
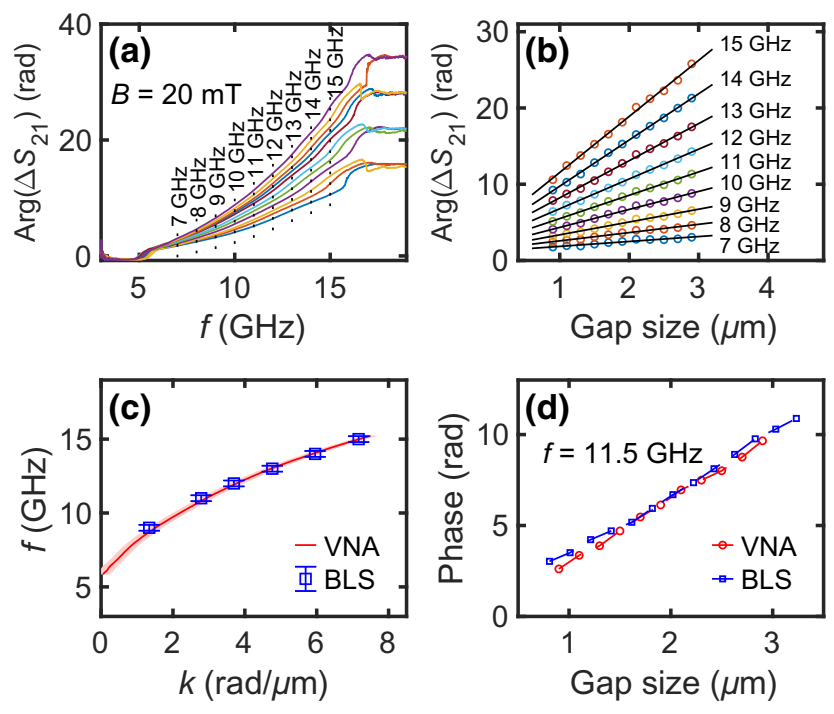

FIG. 3. Extraction of the dispersion relation from the outcomes of variable-gap-propagating-spin-wave-spectroscopy (VGPSWS) experiment on $30-\mathrm{nm} \mathrm{Co}-\mathrm{Fe}-\mathrm{B}$ thin film at $B=$ $20 \mathrm{mT}$ in the $\mathbf{k} \perp \mathbf{B}$ geometry. (a) The unwrapped $\Delta S_{21}$ phases measured over gap widths ranging from $0.9 \mu \mathrm{m}$ (the lowest line) to $2.9 \mu \mathrm{m}$ (the steepest line) with a step of $200 \mathrm{~nm}$. (b) The representative fits of the phase at selected frequencies versus gap size where the slope of the fit yields the desired $k$-vector at that frequency. (c) The dispersion relation extracted for all frequencies within the range with sufficient VG PSWS signal (red line with filled-area error bars) compared with dispersion obtained by phase-resolved BLS (blue squares with error bars). (d) The phase measurements obtained by VGPSWS (red) and phase-resolved BLS (blue) at frequency $f=11.5 \mathrm{GHz}$. 
$k$-vectors against their frequencies, showing the resulting dispersion relation in Fig. 3(c). To confirm the result, we remeasure the same sample using phase-resolved BLS [4], and we find very good agreement within the error bars of each technique. A comparison of the dispersion relations measured by both techniques (VNA in red and BLS in blue) is shown in Fig. 3(c), and a comparison of the measured phase evolution at the frequency of $11.5 \mathrm{GHz}$ is shown in Fig. 3(d).

\section{MEASURED SPIN-WAVE DISPERSION RELATIONS}

Figure 4(a) shows dispersion relations for the same 30$\mathrm{nm}$ Co-Fe-B thin film measured in the $\mathbf{k} \perp \mathbf{B}$ geometry in magnetic fields ranging from 20 to $380 \mathrm{mT}$ with a step of $60 \mathrm{mT}$. The sample is also measured in the $\mathbf{k} \| \mathbf{B}$ geometry, but the measured signal is insufficient to reconstruct the dispersion relation. For all measured fields, it is possible to evaluate the dispersion for $k$-vectors ranging from 0 to $8 \mathrm{rad} / \mu \mathrm{m}$. The upper limit is given by the excitation efficiency [15] of the antenna used (see the blue lines in Fig. 4). By fitting the measured dispersions using the Kalinikos-Slavin model [30], we are able to obtain material parameters of the measured thin films (see the black lines in Fig. 4 for the model fits and the figure caption for the fitting results).

In addition to the 30-nm Co-Fe-B thin film, we also measure 100-nm-thick Co-Fe-B [Fig. 4(b)] and 100-nm-thick YIG [Fig. 4(c)] films to further explore the possibilities of the technique presented. The measurements are performed with different antenna types (stripline, GS, and CPW) to see their influence on the quality of the dispersions obtained, and they are also evaluated for multiple magnetic fields in the $\mathbf{k} \perp \mathbf{B}$ and in $\mathbf{k} \| \mathbf{B}$ geometries. The 100 -nm-thick Co-Fe-B film is measured in fields ranging from 20 to $380 \mathrm{mT}$ with a step of $60 \mathrm{mT}$ at a power of 0 dBm. In Fig. 4(b) we show the dispersion measured with a 500-nm-wide stripline antenna for the $\mathbf{k} \perp \mathbf{B}$ geometry and with a $500-\mathrm{nm}$ coplanar waveguide (the signal and ground line widths, as well as the signal-to-ground gap, are 500 $\mathrm{nm})$ in the $\mathbf{k} \perp \mathbf{B}$ geometry. We are able to obtain a dispersion in the $\mathbf{k} \| \mathbf{B}$ geometry also using a 500-nm-wide stripline antenna but with substantially worse quality due to its lower excitation efficiency. The 100-nm-thick YIG film is measured in the $\mathbf{k} \perp \mathbf{B}$ and $\mathbf{k} \| \mathbf{B}$ geometries in fields ranging from 20 to $200 \mathrm{mT}$ with a step of $20 \mathrm{mT}$ at a power of $-30 \mathrm{dBm}$. In this case, it is possible to obtain a dispersion in both geometries with all types of antennas. Figure 4(c) shows data acquired by GS antennas with gap widths ranging from 1.0 to $3.4 \mu \mathrm{m}$ with a $400-\mathrm{nm}$ step. As in the case of the coplanar waveguide, the dimensions of the GS antennas are $500 \mathrm{~nm}$ (signal and ground line widths and signal-to-ground gap width). The dispersions are plotted for magnetic fields from 20 to $200 \mathrm{mT}$ with a step of $20 \mathrm{mT}$ and the power output set to $-30 \mathrm{dBm}$.

In the dispersion measured on 100-nm-thick Co-Fe-B film, we further identify visible hybridizations at the crossing positions between the $n=0$ fundamental spin-wave mode and $n=1,2,3$ higher-order perpendicular standing spin-wave modes (see Fig. 5). The hybridizations are present in the measured dispersion relation as gap
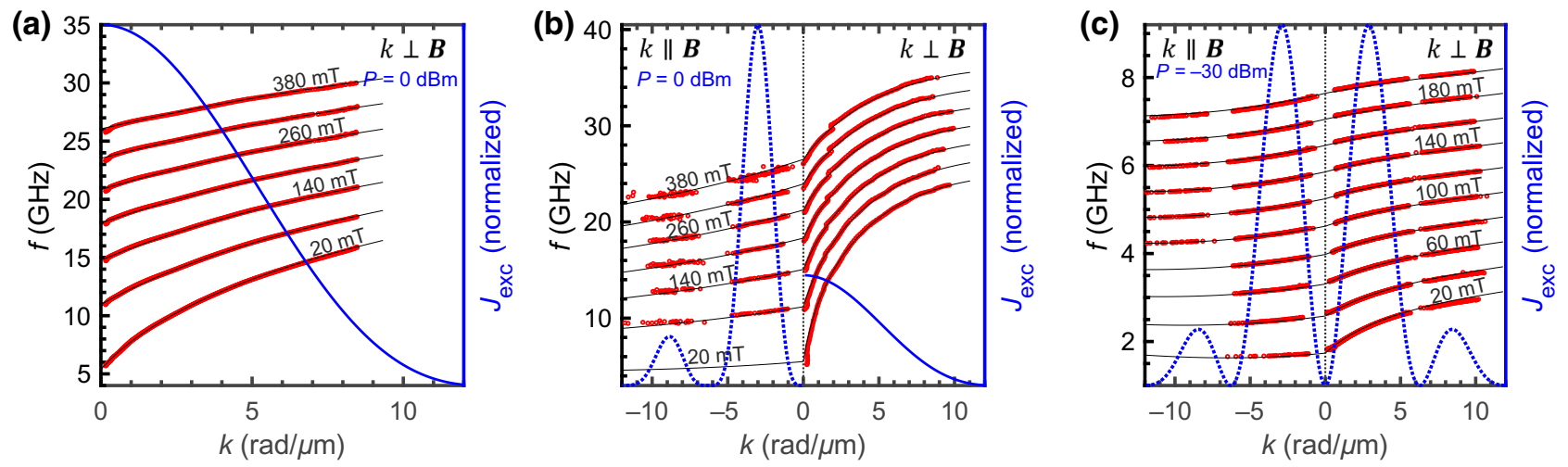

FIG. 4. Examples of VGPSWS dispersion measurements on three different samples. Each panel shows the measured dispersion (red points) compared with the analytical dispersion from the Kalinikos-Slavin model (black lines) together with the normalized excitation spectrum of the antenna used for the measurement (blue lines). For clarity, the data for $\mathbf{k} \perp \mathbf{B}$ are plotted in the domain of positive wave numbers $k$ and the $\mathbf{k} \| \mathbf{B}$ data are presented with negative $k$. Panel (a) shows 30-nm-thick Co-Fe-B measured by the stripline antenna. Panel (b) shows 100-nm-thick Co-Fe-B measured by the CPW (in negative $k$ ) and by the stripline antenna (in positive $k$ ). Panel (c) shows 100-nm-thick YIG measured by the GS antenna. For the Kalinikos-Slavin model we fit the following values of the parameters ( $t$ stands for thickness of the layer): for (a) 30 -nm-thick Co-Fe-B, $M_{s}=1230 \mathrm{kA} / \mathrm{m}\left(\mu_{0} M_{s}=1.54 \mathrm{~T}\right), \gamma / 2 \pi=$ $30.5 \mathrm{GHz} / \mathrm{T}, t=29.0 \mathrm{~nm}$; (b) for $100-\mathrm{nm}$-thick Co-Fe-B, $M_{s}=1310 \mathrm{kA} / \mathrm{m}\left(\mu_{0} M_{s}=1.64 \mathrm{~T}\right), \gamma / 2 \pi=30.1 \mathrm{GHz} / \mathrm{T}$; (c) for $100 \mathrm{~nm}$ YIG, $M_{s}=133 \mathrm{kA} / \mathrm{m}\left(\mu_{0} M_{s}=0.166 \mathrm{~T}\right), \gamma / 2 \pi=28.3 \mathrm{GHz} / \mathrm{T}$. In the fitting procedure, we fix the exchange stiffness $A_{\mathrm{ex}}$ to $14 \mathrm{pJ} / \mathrm{m}$ for Co-Fe-B films and to $3.6 \mathrm{pJ} / \mathrm{m}$ for the YIG film. 

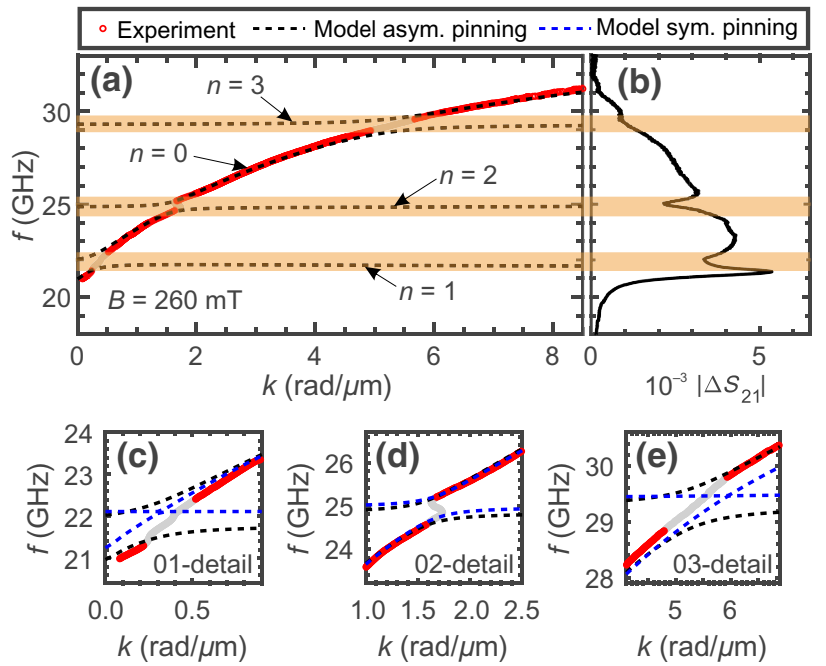

FIG. 5. (a) Measured dispersion relation (red points) for a 100-nm-thick Co-Fe-B layer at $260 \mathrm{mT}$ exhibiting hybridized spin-wave modes, visible mainly at the 02 crossing. The dashed lines represent a numerical model of dispersion with asymmetric surface pinning conditions with parameters $K_{S}=0$ and $1600 \mu \mathrm{J} / \mathrm{m}^{2}$ on the top face and the bottom face of the layer, respectively. (b) Corresponding magnitude of the transmission parameter $\Delta S_{21}$ exhibiting dips at the crossing positions $\left(\left|\Delta S_{21}\right|\right)$ data shown for a gap width of $1.8 \mu \mathrm{m}$. (c)-(e) Details of the 01, 02, and 03 crossings, respectively. Dashed blue lines correspond to the numerical model with symmetric surface pinning conditions $\left(K_{S}=700 \mu \mathrm{J} / \mathrm{m}^{2}\right.$ on both faces).

openings, with a portion of the measured data evolving toward smaller $k$-vectors [see Fig. 5(d) for the mostprominent example]. This part of the dispersion has no physical meaning and is only a result of the data processing described earlier in the text. The hybridizations are also directly visible as dips in the magnitude $\left|\Delta S_{21}\right|$ of the transmission spectra [see Fig. 5(b)].

\section{NUMERICAL MODELING}

To investigate numerically the hybridization between the fundamental mode and perpendicular standing modes, we solve the Landau-Lifshitz equation (LLE) using the finite-element method in the frequency domain. The LLE is linearized and implemented in a finite-element-method solver (COMSOL Multiphysics) as a set of differential equations for the in-plane and the out-of-plane components of magnetization $m_{\|}$and $m_{\perp}$,

$$
\begin{aligned}
& i \omega m_{\|}=-|\gamma| \mu_{0}\left[\left(H_{0}-\frac{2 A_{\mathrm{ex}}}{\mu_{0} M_{s}} \Delta\right) m_{\perp}+M_{s} \partial_{x_{\perp}} \varphi\right], \\
& i \omega m_{\perp}=|\gamma| \mu_{0}\left[\left(H_{0}-\frac{2 A_{\mathrm{ex}}}{\mu_{0} M_{s}} \Delta\right) m_{\|}+M_{s} \partial_{x_{\|}} \varphi\right],
\end{aligned}
$$

together with the equation for the scalar magnetostatic potential $\varphi$ derived from the Maxwell equations in the magnetostatic approximation [31,32]:

$$
\Delta \varphi-\partial_{x_{\|}} m_{\|}-\partial_{x_{\perp}} m_{\perp}=0,
$$

where $x_{\|}$and $x_{\perp}$ denote the directions of the corresponding dynamic components of magnetization. A detailed description of the COMSOL Multiphysics simulation is given in the Appendix.

In contrast to the analytical model of Kalinikos and Slavin, our numerical calculations truly reproduce the spin dispersion relation in the crossover dipolar-exchange regime [2], including the hybridizations between the fundamental spin-wave mode and perpendicular standing spin-wave modes. In our model, we also consider the presence of surface anisotropy $K_{s}$. The anisotropy is expected to be different on the bottom and the top faces of the magnetic layer interfaced with different materials. The surface anisotropy and its asymmetry (between the top face and the bottom face) is responsible for the spin-wave pinning and the strength of the hybridization between fundamental and perpendicular standing modes. The pinning is implemented as the boundary conditions [33]

$$
\begin{aligned}
\partial_{x_{\perp}} m_{\|} & =0, \\
A_{\text {ex }} \partial_{x_{\perp}} m_{\perp}-K_{s} m_{\perp} & =0 .
\end{aligned}
$$

The numerical model (as well as the analytical model of Kalinikos and Slavin) is characterized by a few material parameters. We fix the value of the exchange stiffness $A_{\text {ex }}$ to $14 \mathrm{pJ} / \mathrm{m} \mathrm{[34]} \mathrm{and} \mathrm{the} \mathrm{layer's} \mathrm{thickness} d$ to the nominal value of $100 \mathrm{~nm}$. The values of saturation magnetization $M_{s}=1275 \mathrm{kA} / \mathrm{m}$ and the gyromagnetic ratio $|\gamma| / 2 \pi=$ $30.8 \mathrm{GHz} / \mathrm{T}$ were selected to fit the ferromagnetic resonance frequency (i.e., the frequency of the fundamental mode at $k=0$ ) and the slope of the dispersion for the fundamental mode.

The surface anisotropy $K_{s}$ determines the spin-wave pinning and therefore is important both for the quantization (and the frequencies) of the spin-wave modes and the strength of the hybridization between them. In the absence of surface anisotropy, the magnetization dynamics on the surfaces is released due to exchange interaction, and the spin wave has antinodes there $\left(\partial_{x_{\perp}} m_{\perp}=0\right)$, whereas positive (negative) values of $K_{s}$ increase (decrease) the amplitude of spin-wave precession at the surfaces. Therefore, by playing with the surface anisotropy, and in particular by introducing asymmetry in their profiles by differentiating $K_{s}$ (and the strength of the pinning) on the top and bottom surfaces of the magnetic layer, we can influence the cross section between different modes and modify their hybridization.

To obtain the proper values of the frequencies of perpendicular standing modes, we do not need to reduce the thickness below the nominal value of $100 \mathrm{~nm}$ but we have to introduce a nonzero $K_{s}$ instead, which seems 
to be a more-realistic approach. The experimental data show that hybridization of the fundamental mode with the perpendicular standing modes is observed for the perpendicular standing modes quantized with both an even number $(n=2)$ and an odd number $(n=1,3)$ of nodes across the layer. The effect for $n=1,3$ is hardly visible in the dispersion relation [Figs. 5(c) and 5(e)] but is quite distinctive in the measurement of the transmission amplitude [Fig. 5(b)]. These results indicate that the asymmetric pinning (and the different values of surface anisotropy on both faces of the Co-Fe-B layer) must be considered to obtain less-symmetric profiles of spin-wave modes across the thickness of the layer, which, in turn, gives the nonzero cross sections between the fundamental mode and perpendicular standing modes with odd numbers of nodes. There is always some degree of freedom in choosing the values of $K_{s}$ on both faces; therefore, we decide to consider the simplest case, where $K_{s}=0$ and $1600 \mu \mathrm{J} / \mathrm{m}^{2}$ at the top (interface with a vacuum) and bottom (interface with GaAs), respectively. With these values, we succeed in the induction of the hybridization of the fundamental mode $(n=0)$ with the first $(n=1)$ and third $(n=3)$ perpendicular standing modes, characterized by a width comparable to the widths of the dips in the transmission spectra $\Delta S_{12}$ [marked by orange stripes in Fig. 5(b)]. In the case of symmetric pinning (we take $K_{s}=700 \mu \mathrm{J} / \mathrm{m}^{2}$ on both faces), we do not observe hybridization with the first $(n=1)$ and third $(n=3)$ perpendicular standing modes [see the dashed blue lines in Figs. 5(c) and 5(e)]. This can be intuitively understood when we notice that, for symmetric pinning, the cross section of the fundamental mode (i.e., the even mode without any node inside the layer) with perpendicularly standing modes with an odd number of nodes (i.e., the modes with $1,3,5, \ldots$ nodes inside the layer) is zero or very small.

\section{ADDITIONAL DATA ANALYSIS AND DISCUSSION}

From the measured dispersion relations it is possible to extract also other parameters important for spinwave propagation. The group velocity $v_{g}$ can be calculated as the numerical derivative of the dispersion $v_{g}=2 \pi d f / d k$, and the lifetime $\tau$ can be obtained as the numerical derivative of the field-dependent dispersion [31]: $\tau=\left(\alpha \omega \partial \omega / \partial \omega_{B}\right)^{-1}=\left(\alpha 4 \pi^{2} f / \gamma \partial f / \partial B\right)^{-1}$, where $\omega_{B}=\gamma B$ and $\alpha$ is a damping constant.

The decay length $\delta$ can be then calculated by multiplying the group velocity and the lifetime $\delta=v_{g} \tau$. In Fig. 6(a), we plot the decay length obtained by the abovedescribed procedure (orange line) and compare it with the decay length obtained with the more-traditional approach, i.e., by fitting the exponential decay of the magnitude of the $\Delta S_{21}$ signal (blue line). Here, the decay length $\delta$ is obtained by fitting [see Fig. 6(b) for representative fits] the formula (a)

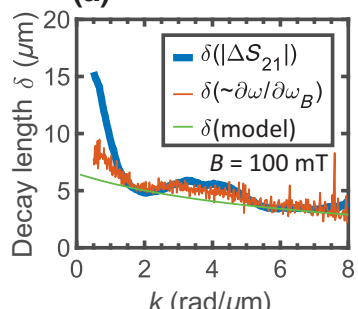

(c)
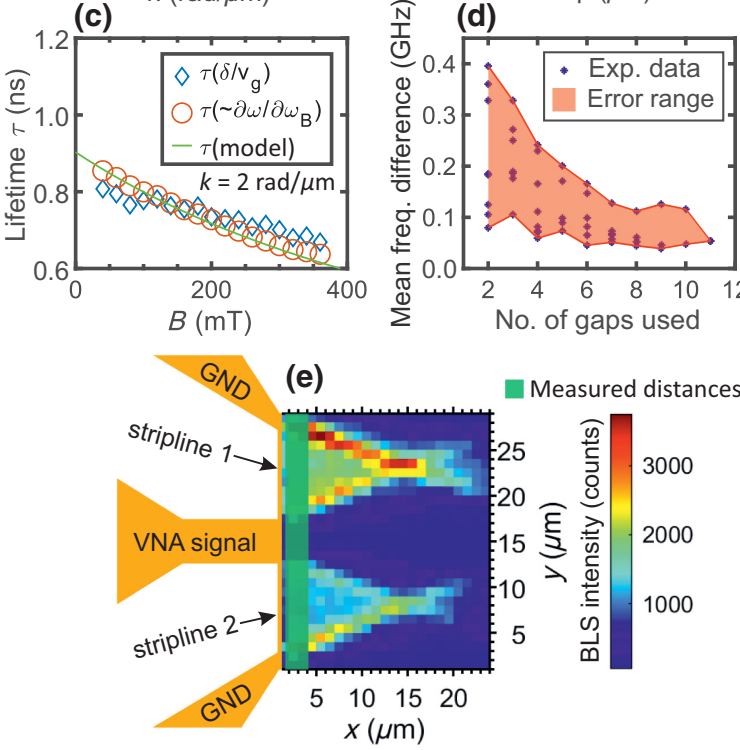

FIG. 6. (a)-(c),(e) Determination of decay length and lifetime from VGPSWS measurements of 30-nm-thick Co-Fe-B film and and (d) impact of the number of gaps on the quality of the result. (a) The blue line shows the spin-wave decay lengths obtained directly from exponential fits, the orange line shows the decay length calculated by multiplication of the lifetime and the group velocity, and the green line is calculated from the KalinikosSlavin model. (b) Representative exponential fits of the $\Delta S_{21}$ magnitude that were used to obtain the spin-wave propagation length. (c) Spin-wave lifetime extracted from the derivative of the field-dependent dispersion with use of $\alpha=0.075$ (orange circles), calculated by our dividing the propagation length (from the $\Delta S_{21}$ magnitude) by the numerically-calculated group velocity (blue diamonds) and calculated from the Kalinikos-Slavin model (green line). (d) Mean frequency difference of the dispersion obtained from the reduced number of gap widths compared with the analytical fit of the dispersion obtained from the complete set of 11 measured points. Blue points represent different combinations of gaps and the red area represents the difference spread. (e) Spin-wave caustics caused by the finite length of the antenna, measured on 100-nm-thick Co-Fe-B film by BLS with an excitation frequency of $11 \mathrm{GHz}$ in an external magnetic field of $26 \mathrm{mT}$. Caustics can destroy the phase coherence of propagating spin waves. All data presented are collected at propagation distances (gap widths) between 0.9 and $2.9 \mu \mathrm{m}$ (green region) to avoid the negative effects of the formation of caustics. GND, ground.

$\left|\Delta S_{21}\right|=I e^{-g / \delta}$ in logarithmic form $\ln \left|\Delta S_{21}\right|=\ln I-$ $g / \delta$, where $g$ is the gap distance and $I$ is a free parameter proportional to the signal strength. In Fig. 6(c), we 
plot the field-dependent lifetime evaluated with the decay length obtained from the derivative of the field-dependent dispersion $\left(\partial \omega / \partial \omega_{B}\right.$, orange circles) and from fitting of the exponential decay of the magnitude of the $\Delta S_{21}$ signal (blue diamonds). Both approaches give roughly the same results for both the decay length and the lifetime, but the decay lengths obtained by the $\partial \omega / \partial \omega_{B}$ approach agree better with the analytical model [see Fig. 6(a), green line]. The lower quality of the data obtained from fitting the exponential decay of the magnitude of the $\Delta S_{21}$ signal is caused by the limitation of our experimental arrangement. Because of the finite length of the excitation antenna, spinwave caustics can form [35]. The phase in the caustic beam is spatially incoherent [36] and the focusing effect [37] causes modulation of the spin-wave intensity along the propagation direction. An example of such caustics measured by BLS microscopy on 100-nm-thick Co-Fe-B film is shown in Fig. 6(e). The formation of caustics depends on the material and on the exact geometry of the experiment. Without imaging techniques such as BLS or the time-resolved magneto-optical Kerr effect (MOKE), it may be difficult to ensure that the caustics do not distort the experiment. A safe precaution, which we use through our experiments, is to measure at propagation distances that are sufficiently small with respect to the antenna length. In our experiments, we use an antenna length of $10 \mu \mathrm{m}$, and the maximum measured propagation distance is $2.9 \mu \mathrm{m}$ [see the green region in Fig. 6(e)]. For phase-resolved measurements, such as evaluation of dispersions, the short propagation distance is sufficient. On the other hand, the change in signal at a propagation distance of $2.9 \mu \mathrm{m}$ is not sufficient to obtain a fully reliable fit of the exponential decay of the $\Delta S_{21}$ magnitude and at longer propagation distances the measurements are distorted by caustics.

We also analyze how the number of measured propagation distances (gaps) affects the reliability of the dispersion obtained. We take the data measured for 11 gap widths in total and then use different combinations of a reduced number of gap widths (starting from 2 up to 10) to evaluate the dispersion. The dispersion obtained from the reduced number of gap widths is then compared with the analytical fit (with use of the Kalinikos-Slavin model [30]) of the dispersion obtained from fitting of the complete set of 11 measured points. As shown in Fig. 6(d), the mean frequency difference from the reference dispersion increases to a maximum of $400 \mathrm{MHz}$ when a combination of just two gap widths is used. This maximum quickly decreases to $200 \mathrm{MHz}$ for five gap widths and then stays around $100 \mathrm{MHz}$ for combinations of six or more gaps.

As can be seen from the data presented, the variable-gap approach allows the reconstruction of the spin-wave dispersion relation, including detailed features that might be used for analysis of the spin-wave system. To obtain the highest-possible data quality, we need to fabricate multiple pairs of antennas where the gap width and the step in the gap width (an increase of the gap between two pairs of antennas) must be optimized for each experiment. This approach does not require precise knowledge of the excitation-spot location (phase origin) because it does not affect the fit's slope. It is essential to know only the relative differences between the propagation distances, i.e., the gap-width step, which can be easily measured (and precisely fabricated by electron-beam lithography).

The antenna design is sample specific and needs to be tailored to fulfill experimental requirements. The antenna's type and geometry must be able to excite the expected $k$ vector range with sufficient efficiency.

The gap widths need to be in the optimum range considering the formation of caustics and the spin-wave decay length for the given material and geometry. For example, for 30-nm-thick Co-Fe-B in the $\mathbf{k} \| \mathbf{B}$ geometry at $k=5 \mathrm{rad} / \mu \mathrm{m}$, we calculate decay lengths of 0.03 and $0.45 \mu \mathrm{m}$ for magnetic fields of 20 and $200 \mathrm{mT}$, respectively. Here, the quality of the measured data, even for the smallest fabricated gap distance of $1 \mu \mathrm{m}$, is not sufficient to evaluate dispersion; thus, this geometry is not presented in Fig. 4(a).

Before the data are fitted as plotted in Fig. 3(b), the phase needs to be correctly unwrapped. In the case of stripline antennas with a continuous excitation spectrum, it is possible to achieve correct unwrapping even when the phases for neighboring gap widths at the same frequency are higher than $\pi$ rad by unwrapping the phase in the frequency spectrum [Fig. 3(a)]. This is because the frequency step size of the VNA is usually small and the phase shift of the neighboring points is always smaller than $\pi \mathrm{rad}$. On the other hand, the safest approach is to unwrap the phases when plotted against the gap size [Fig. 3(b)]. In this case, the phase change of neighboring points must be smaller than $\pi \mathrm{rad}$. This is necessary for antenna types with discrete excitation spectra (i.e., CPW, GS, ladders [45], or meanders [46]).

The step in the gap width defines the maximum $k$-vector for which the dispersion can be measured. On the other hand, small $k$-vectors may have a phase change that is too small over a short step in the gap width. If we require an accurate fitting of small $k$-vectors, the step in the gap width should be large enough to provide sufficient phase change (we suggest at least $1 \mathrm{rad}$ ) while respecting the decay length.

\section{METHOD COMPARISON}

In Table I, we compare variable-gap propagating spinwave spectroscopy (VGPSWS) with other experimental techniques used to obtain spin-wave dispersion relations. To get detailed dispersion, one needs to have high resolution in both the $k$-vector and the frequency. Optical and $\mathrm{x}$-ray techniques have low frequency resolution, which is typically limited to hundreds of megahertz. In the 
TABLE I. Comparison of experimental techniques used for measurement of spin-wave dispersion relations.

\begin{tabular}{lcccccc}
\hline \hline & $\begin{array}{c}\text { Lateral } \\
\text { resolution } \\
(\mathrm{nm})\end{array}$ & $\begin{array}{c}k \\
\text { resolution } \\
(\mathrm{rad} / \mu \mathrm{m})\end{array}$ & $\begin{array}{c}\text { Frequency } \\
\text { resolution } \\
(\mathrm{Hz})\end{array}$ & $\begin{array}{c}\text { Phase } \\
\text { extraction }\end{array}$ & $\begin{array}{c}\text { Maximum } k \\
(\mathrm{rad} / \mu \mathrm{m})\end{array}$ & $\begin{array}{c}\text { Experimental } \\
\text { geometries }\end{array}$ \\
\hline VGPSWS (this work) & $\ldots$ & $<0.1^{\mathrm{a}}$ & 1 & Yes & $10^{\mathrm{b}}$ & MS, BV (FV possible) \\
PSWS [17,22] & $\ldots$ & $2^{\mathrm{b}}$ & 1 & No & $10^{\mathrm{b}}$ & $\begin{array}{c}\text { MS, BV, FV } \\
\text { MOKE [38,39] }\end{array}$ \\
Conventional BLS [2,40] & 500 & $<0.1^{\mathrm{a}}$ & $1 \times 10^{8 \mathrm{c}}$ & Yes & 10 & BV, MS \\
Phase-resolved BLS [4,41,42] & 10000 & 0.5 & $1 \times 10^{8}$ & No & 23.6 & BV, MS \\
STXM [43,44] & 250 & $<0.1^{\mathrm{a}}$ & $1 \times 10^{8 \mathrm{c}}$ & Yes & 7 & BV, MS \\
\hline \hline
\end{tabular}

BV, backward volume spin waves; FV, forward volume spin waves; MS, magnetostatic surface spin waves.

${ }^{\text {a }}$ The $k$-resolution can be tailored to the magnetic system under study by appropriate design of the experimental geometry.

${ }^{\mathrm{b}}$ Excitation limited.

${ }^{\mathrm{c}}$ The frequency resolution in these techniques is limited by the acquisition time.

case of conventional BLS, the resolution is determined directly by the Fabry-Perot interferometer [47]. The optical techniques using microwave excitation, i.e., MOKE, phase-resolved BLS, and scanning transmission x-ray microscopy (STXM), are limited by slow signal acquisition. They do not allow the capturing of the required span of frequencies with high resolution in a reasonable time. The propagating-spin-wave-spectroscopy technique, which uses known positions of the excitation peaks of the CPW antenna, can capture the data with high frequency resolution. However, the $k$-resolution is limited by the finite widths of the peaks in the excitation spectra of the CPW antennas used, which in turn affect also the frequency resolution.

A big advantage of the VGPSWS technique is that it does not require direct optical access to the sample, making the method very suitable for, for example, experiments at ultralow temperatures. In addition, compared with other techniques for measurement of the spin-wave dispersion relation, VGPSWS can achieve high frequency resolution and high $k$-vector resolution at the same time. This combination allows the capturing of smooth dispersion curves over the span of all accessible $k$-vectors and the fast acquisition times allow the experiment to be repeated in magnetic field of multiple strengths and orientations. The full set of field-dependent dispersion curves measured for the $\mathbf{k} \perp \mathbf{B}$ and $\mathbf{k} \| \mathbf{B}$ geometries present a robust four-dimensional $(f, k, B$, and angle between $\mathbf{k}$ and B) dataset that can be further evaluated, and all essential material and spin-wave parameters can be extracted from it.

A disadvantage of this method is the need for a set of antennas with multiple gap widths on top of the sample. The other techniques can obtain the dispersion without an antenna (conventional BLS), with one excitation antenna (MOKE, phase-resolved BLS, and STXM), or with a pair of excitation and detection antennas (PSWS). However, the need for multiple antennas may be overcome in the future by use of freestanding positionable antennas [48].

\section{CONCLUSION}

In conclusion, we present a method of extraction of a high-quality spin-wave dispersion relation from PSWS measurements performed over several propagation distances. We demonstrate this technique on $\mathrm{Co}-\mathrm{Fe}-\mathrm{B}$ and YIG thin films measured in the $\mathbf{k} \perp \mathbf{B}$ and $\mathbf{k} \| \mathbf{B}$ geometries. The results on Co-Fe-B thin film are verified by phase-resolved BLS measurement, showing good agreement. When compared with phase-resolved BLS, the VNA-based method provides more frequency measurement points in a shorter acquisition time. Fine-detail measurement capability is demonstrated by the measurement and analysis of hybridized modes acquired on 100-nmthick Co-Fe-B thin film, revealing asymmetric surface pinning and the values of the pinning parameters on both interfaces of the magnetic layer. The all-electric nature of this method makes it very suitable for characterization of cryogenic and quantum magnonics systems and materials.

\section{ACKNOWLEDGMENTS}

The work was supported by MEYS CR (project CZ.02.2.69/0.0/0.0/19_073/0016948). CzechNanoLab project LM2018110 is gratefully acknowledged for financial support of the measurements and sample fabrication at CEITEC Nano Research Infrastructure. O.W. was supported by the Brno PhD Talent Scholarship. J.W.K. and M.K. acknowledge the support of the National Science Centre, Poland, for the projects UMO2020/37/B/ST3/03936 and UMO-2020/39/O/ST5/02110. A.V.C. acknowledges the Austrian Science Fund (FWF) for support through Grant No. I 4696-N (Nano-YIG). O.V.D. acknowledges the Austrian Science Fund (FWF) for support through Grant No. I 4889 (CurviMag). C.D. gratefully acknowledges financial support from the Deutsche Forschungsgemeinschaft (DFG, German Research Foundation) - 271741898. 


\section{APPENDIX: DETAILS OF THE NUMERICAL SIMULATIONS IN COMSOL MULTIPHYSICS}

The geometry consists of magnetic material with thickness of $100 \mathrm{~nm}$ and nonmagnetic material with thickness on the order of the wavelength of the smallest nonzero wave vector calculated (we use $5 \mu \mathrm{m}$ ), which is positioned over and under the magnetic material. The width of all elements is $10 \pi \mathrm{nm}$. The LLE in the linearized form [Eqs. (1) and (2)] is implemented using Coefficient Form PDE for two dependent variables $m_{\|}$and $m_{\perp}$. The equation is solved only in the magnetic materials. The surface anisotropy [Eqs. (4) and (5)] is implemented with use of the Flux/Source boundary condition. It requires only the implementation of the surface anisotropy term as the exchange term is implemented automatically with the LLE. Another Coefficient Form PDE is used to implement Eq. (3), separately for magnetic and nonmagnetic material (the latter using Poisson's Equation). The Dirichlet Boundary Condition for zeroing of the magnetic potential $\varphi$ is added at the top and bottom boundaries of the whole geometry. To simulate the effect of the infinite layer, we use Floquet boundary conditions. We add it using the Pointwise Constraint node, which is added on the right boundary of the geometry with use of the constraint expression $P_{\mathrm{LB}}-P_{\mathrm{RB}} \exp (i k \pi / a)=0$, where $P$ is one of the calculated magnetic parameters $\left(m_{\|}, m_{\perp}\right.$, or $\left.\varphi\right), \mathrm{LB}$ and RB are the left-boundary position and the right-boundary position, respectively, $k$ is a wave vector, and $a$ is the width of the structure. Pointwise Constraint has to be applied to all three calculated magnetic parameters. The values from the left boundary are exported to Pointwise Constraint with use of Linear Extrusion coupling. It has to be applied separately for magnetic materials (used in $m_{\|}$and $m_{\perp}$ ) and the whole geometry (used in $\varphi$ ). The triangular mesh has a maximum element size of $5 \mathrm{~nm}$ in the magnetic material. The study is performed with use of Eigenfrequency solver with Parametric Sweep of the wave-vector values in the experimental range.

[1] B. Dieny, et al., Opportunities and challenges for spintronics in the microelectronics industry, Nat. Electron. 3, 446 (2020).

[2] S. Tacchi, R. Silvani, G. Carlotti, M. Marangolo, M. Eddrief, A. Rettori, and M. G. Pini, Strongly hybridized dipole-exchange spin waves in thin $\mathrm{Fe}-\mathrm{N}$ ferromagnetic films, Phys. Rev. B 100, 104406 (2019).

[3] C. Mathieu, J. Jorzick, A. Frank, S. O. Demokritov, A. N. Slavin, B. Hillebrands, B. Bartenlian, C. Chappert, D. Decanini, F. Rousseaux, and E. Cambril, Lateral Quantization of Spin Waves in Micron Size Magnetic Wires, Phys. Rev. Lett. 81, 3968 (1998).

[4] K. Vogt, H. Schultheiss, S. J. Hermsdoerfer, P. Pirro, A. A. Serga, and B. Hillebrands, All-optical detection of phase fronts of propagating spin waves in a Ni81Fe19 microstripe, Appl. Phys. Lett. 95, 182508 (2009).
[5] V. E. Demidov, S. Urazhdin, and S. O. Demokritov, Control of spin-wave phase and wavelength by electric current on the microscopic scale, Appl. Phys. Lett. 95, 262509 (2009).

[6] D. Lachance-Quirion, S. P. Wolski, Y. Tabuchi, S. Kono, K. Usami, and Y. Nakamura, Entanglement-based single-shot detection of a single magnon with a superconducting qubit, Science 367, 425 (2020).

[7] O. V. Dobrovolskiy, R. Sachser, T. Brächer, T. Böttcher, V. V. Kruglyak, R. V. Vovk, V. A. Shklovskij, M. Huth, B. Hillebrands, and A. V. Chumak, Magnon-fluxon interaction in a ferromagnet/superconductor heterostructure, Nat. Phys. 15, 477 (2019).

[8] I. A. Golovchanskiy, N. N. Abramov, V. S. Stolyarov, V. V. Bolginov, V. V. Ryazanov, A. A. Golubov, and A. V. Ustinov, Ferromagnet/Superconductor hybridization for magnonic applications, Adv. Funct. Mater. 28, 1802375 (2018).

[9] M. Bailleul, D. Olligs, C. Fermon, and S. O. Demokritov, Spin waves propagation and confinement in conducting films at the micrometer scale, Europhys. Lett. (EPL) 56, 741 (2001).

[10] T. Devolder, G. Talmelli, S. M. Ngom, F. Ciubotaru, C. Adelmann, and C. Chappert, Measuring the dispersion relations of spin wave bands using time-of-flight spectroscopy, Phys. Rev. B 103, 214431 (2021).

[11] F. Ciubotaru, T. Devolder, M. Manfrini, C. Adelmann, and I. P. Radu, All electrical propagating spin wave spectroscopy with broadband wavevector capability, Appl. Phys. Lett. 109, 012403 (2016).

[12] K. Yamanoi, S. Yakata, T. Kimura, and T. Manago, Spin wave excitation and propagation properties in a permalloy film, Jpn. J. Appl. Phys. 52, 083001 (2013).

[13] U. K. Bhaskar, G. Talmelli, F. Ciubotaru, C. Adelmann, and T. Devolder, Backward volume vs Damon-Eshbach: A traveling spin wave spectroscopy comparison, J. Appl. Phys. 127, 033902 (2020).

[14] M. Bailleul, D. Olligs, and C. Fermon, Propagating spin wave spectroscopy in a permalloy film: A quantitative analysis, Appl. Phys. Lett. 83, 972 (2003).

[15] V. Vlaminck and M. Bailleul, Spin-wave transduction at the submicrometer scale: Experiment and modeling, Phys. Rev. B 81, 014425 (2010).

[16] P. Gruszecki, M. Kasprzak, A. E. Serebryannikov, M. Krawczyk, and W. Śmigaj, Microwave excitation of spin wave beams in thin ferromagnetic films, Sci. Rep. 6, 22367 (2016).

[17] H. Qin, S. J. Hamalainen, K. Arjas, J. Witteveen, and S. van Dijken, Propagating spin waves in nanometer-thick yttrium iron garnet films: Dependence on wave vector, magnetic field strength, and angle, Phys. Rev. B 98, 224422 (2018).

[18] H. Yu, O. d'Allivy Kelly, V. Cros, R. Bernard, P. Bortolotti, A. Anane, F. Brandl, R. Huber, I. Stasinopoulos, and D. Grundler, Magnetic thin-film insulator with ultra-low spin wave damping for coherent nanomagnonics, Sci. Rep. 4, 6848 (2014).

[19] M. Collet, O. Gladii, M. Evelt, V. Bessonov, L. Soumah, P. Bortolotti, S. O. Demokritov, Y. Henry, V. Cros, M. Bailleul, V. E. Demidov, and A. Anane, Spin-wave propagation in ultra-thin YIG based waveguides, Appl. Phys. Lett. 110, 092408 (2017).

[20] O. V. Dobrovolskiy, R. Sachser, S. A. Bunyaev, D. Navas, V. M. Bevz, M. Zelent, W. Smigaj, J. Rychly, M. Krawczyk, 
R. V. Vovk, M. Huth, and G. N. Kakazei, Spin-wave phase inverter upon a single nanodefect, ACS Appl. Mater. Interf. 11, 17654 (2019).

[21] A. Krysztofik, H. Głowiński, P. Kuświk, S. Zietek, L. E. Coy, J. N. Rychły, S. Jurga, T. W. Stobiecki, and J. Dubowik, Characterization of spin wave propagation in (1 11) YIG thin films with large anisotropy, J. Phys. D: Appl. Phys. 50, 235004 (2017).

[22] M. Sushruth, M. Grassi, K. Ait-Oukaci, D. Stoeffler, Y. Henry, D. Lacour, M. Hehn, U. Bhaskar, M. Bailleul, T. Devolder, and J.-P. Adam, Electrical spectroscopy of forward volume spin waves in perpendicularly magnetized materials, Phys. Rev. Res. 2, 043203 (2020).

[23] J. Chen, F. Heimbach, T. Liu, H. Yu, C. Liu, H. Chang, T. Stückler, J. Hu, L. Zeng, Y. Zhang, Z. Liao, D. Yu, W. Zhao, and $\mathrm{M}$. Wu, Spin wave propagation in perpendicularly magnetized nm-thick yttrium iron garnet films, J. Magn. Magn. Mater. 450, 3 (2018).

[24] J. Béjanin, T. McConkey, J. Rinehart, C. Earnest, C. McRae, D. Shiri, J. Bateman, Y. Rohanizadegan, B. Penava, P. Breul, S. Royak, M. Zapatka, A. Fowler, and M. Mariantoni, Three-Dimensional Wiring for Extensible Quantum Computing: The Quantum Socket, Phys. Rev. Appl. 6, 044010 (2016).

[25] C. Dubs, O. Surzhenko, R. Linke, A. Danilewsky, U. Brückner, and J. Dellith, Sub-micrometer yttrium iron garnet LPE films with low ferromagnetic resonance losses, J. Phys. D: Appl. Phys. 50, 204005 (2017).

[26] C. Dubs, O. Surzhenko, R. Thomas, J. Osten, T. Schneider, K. Lenz, J. Grenzer, R. Hubner, and E. Wendler, Low damping and microstructural perfection of sub-40nm-thin yttrium iron garnet films grown by liquid phase epitaxy, Phys. Rev. Mater. 4, 024416 (2020).

[27] K. Zakeri, J. Lindner, I. Barsukov, R. Meckenstock, M. Farle, U. von Horsten, H. Wende, W. Keune, J. Rocker, S. S. Kalarickal, K. Lenz, W. Kuch, K. Baberschke, and Z. Frait, Spin dynamics in ferromagnets, Gilbert damping and two-magnon scattering, Phys. Rev. B 76, 104416 (2007).

[28] T. Schneider, A. A. Serga, T. Neumann, B. Hillebrands, and M. P. Kostylev, Phase reciprocity of spin-wave excitation by a microstrip antenna, Phys. Rev. B 77, 214411 (2008).

[29] K. Sekiguchi, K. Yamada, S. M. Seo, K. J. Lee, D. Chiba, K. Kobayashi, and T. Ono, Nonreciprocal emission of spinwave packet in FeNi film, Appl. Phys. Lett. 97, 022508 (2010).

[30] B. A. Kalinikos and A. N. Slavin, Theory of dipoleexchange spin wave spectrum for ferromagnetic films with mixed exchange boundary conditions, J. Phys. C: Solid State Phys. 19, 7013 (1986).

[31] D. D. Stancil and A. Prabhakar, Spin Waves (Springer US, Boston, MA, 2009).

[32] J. Rychły and J. W. Kłos, Spin wave surface states in 1D planar magnonic crystals, J. Phys. D: Appl. Phys. 50, 164004 (2017).

[33] G. Rado and J. Weertman, Spin-wave resonance in a ferromagnetic metal, J. Phys. Chem. Solids 11, 315 (1959).

[34] J. Cho, J. Jung, K.-E. Kim, S.-I. Kim, S.-Y. Park, M.-H. Jung, and C.-Y. You, Effects of sputtering Ar gas pressure in the exchange stiffness constant of Co40Fe40B20 thin films, J. Magn. Magn. Mater. 339, 36 (2013).
[35] T. Schneider, A. A. Serga, A. V. Chumak, C. W. Sandweg, S. Trudel, S. Wolff, M. P. Kostylev, V. S. Tiberkevich, A. N. Slavin, and B. Hillebrands, Nondiffractive Subwavelength Wave Beams in a Medium with Externally Controlled Anisotropy, Phys. Rev. Lett. 104, 197203 (2010).

[36] H. S. Korner, J. Stigloher, and C. H. Back, Excitation and tailoring of diffractive spin-wave beams in NiFe using nonuniform microwave antennas, Phys. Rev. B 96, 100401(R) (2017).

[37] V. Veerakumar and R. E. Camley, Magnon focusing in thin ferromagnetic films, Phys. Rev. B 74, 214401 (2006).

[38] R. Dreyer, N. Liebing, E. R. J. Edwards, A. Muller, and G. Woltersdorf, Spin-wave localization and guiding by magnon band structure engineering in yttrium iron garnet, Phys. Rev. Mater. 5, 064411 (2021).

[39] H. Qin, R. B. Hollander, L. Flajsman, F. Hermann, R. Dreyer, G. Woltersdorf, and S. van Dijken, Nanoscale magnonic Fabry-Pérot resonator for low-loss spin-wave manipulation, Nat. Commun. 12, 2293 (2021).

[40] T. Sebastian, K. Schultheiss, B. Obry, B. Hillebrands, and H. Schultheiss, Micro-focused brillouin light scattering: Imaging spin waves at the nanoscale, Front. Phys. 3, 35 (2015).

[41] L. Flajsman, K. Wagner, M. Vanatka, J. Gloss, V. Krizakova, M. Schmid, H. Schultheiss, and M. Urbanek, Zerofield propagation of spin waves in waveguides prepared by focused ion beam direct writing, Phys. Rev. B 101, 014436 (2020).

[42] O. Wojewoda, T. Hula, L. Flajsman, M. Vanatka, J. Gloss, J. Holobradek, M. Stano, S. Stienen, L. Körber, K. Schultheiss, M. Schmid, H. Schultheiss, and M. Urbanek, Propagation of spin waves through a Néel domain wall, Appl. Phys. Lett. 117, 022405 (2020).

[43] F. Groß, N. Träger, J. Förster, M. Weigand, G. Schütz, and J. Gräfe, Nanoscale detection of spin wave deflection angles in permalloy, Appl. Phys. Lett. 114, 012406 (2019).

[44] J. Förster, S. Wintz, J. Bailey, S. Finizio, E. Josten, C. Dubs, D. A. Bozhko, H. Stoll, G. Dieterle, N. Träger, J. Raabe, A. N. Slavin, M. Weigand, J. Gräfe, and G. Schütz, Nanoscale $\mathrm{X}$-ray imaging of spin dynamics in yttrium iron garnet, J. Appl. Phys. 126, 173909 (2019).

[45] W. Bang, M. B. Jungfleisch, J. Lim, J. Trossman, C. C. Tsai, A. Hoffmann, and J. B. Ketterson, Excitation of the three principal spin waves in yttrium iron garnet using a wavelength-specific multi-element antenna, AIP Adv. 8, 056015 (2018).

[46] J. Lucassen, C. F. Schippers, L. Rutten, R. A. Duine, H. J. M. Swagten, B. Koopmans, and R. Lavrijsen, Optimizing propagating spin wave spectroscopy, Appl. Phys. Lett. 115, 012403 (2019).

[47] F. Scarponi, S. Mattana, S. Corezzi, S. Caponi, L. Comez, P. Sassi, A. Morresi, M. Paolantoni, L. Urbanelli, C. Emiliani, L. Roscini, L. Corte, G. Cardinali, F. Palombo, J. R. Sandercock, and D. Fioretto, High-Performance Versatile Setup for Simultaneous Brillouin-Raman Microspectroscopy, Phys. Rev. X 7, 031015 (2017).

[48] T. Hache, M. Vanatka, L. Flajsman, T. Weinhold, T. Hula, O. Ciubotariu, M. Albrecht, B. Arkook, I. Barsukov, L. Fallarino, O. Hellwig, J. Fassbender, M. Urbanek, and H. Schultheiss, Freestanding Positionable MicrowaveAntenna Device for Magneto-Optical Spectroscopy Experiments, Phys. Rev. Appl. 13, 054009 (2020). 